\title{
Concepts et Innovations
}

\section{Évaluation d'un enseignement concernant la relation médecin-patient auprès des étudiants de deuxième et troisème annéess des études médicales à la faculté de médecine de Nantes}

\author{
Angélique BONNAUD*, Pierre POTIIER*, Jacques H. BARRIER*, Gérard DABOUIS*, \\ Bernard PLANCHON** et Alain MOUZARD*
}

\begin{abstract}
Résumé Contexte : U n nouvel enseignement concernant la relation médecin-patient a été mis en place de manière exploratoi re auprès des éudiants de deuxième et trois ème années de médecine à la facultéde $\mathrm{N}$ antes; une évaluation dece programme a ééréalisééeauprès desétudiants et des enseignants. But : II sagi ssait d'apprécie l'intérêt et la pertinencepédagogique de cet enseignement. M éthode : N ous avons utilisé des échelles d'appréciations auprès des étudiants et un questionnaire qualitatif auprès des enseignants. Résultats : Les données quantitatives et qualitatives recueillies témoignent d'une appréciation relativement positive de l'enseignement avec le souhait qu'il soit poursuivi durant le second cyde du cursus Conclusion : Compte tenu des limites liéesà son caractèrenovateur et exploratoire, le nouvel enseignement concernant la relation médecin-patient semble être apprécié sil es intégré aux stages qui permettent la contextualisation des connaissances et si l'objectif, à terme, et d'aboutir à l'acquistion d'habiletés adaptés à chaque situation, unique et spécifique du colloque singulier.
\end{abstract}

Mots dés Enseignement de la relation médecin-patient ; évaluation par les étudiants; évaluation par les enseignants; échelles d'appréciation.

\begin{abstract}
An experimental teaching method dedicated to the doctor-patient relationship was initiated at the faculty of medicine in $\mathrm{N}$ antes ( $\mathrm{F}$ rance), followed by an evaluation by second and third year medical students and the teachers. $\mathbf{O}$ bjective: The aim was to assess both the global interest and the pedagogical relevance of this teaching. Method: We used scale estimation for student rating and qualitative questionnaire for teacher rating. Results: Showed relatively sati fying appreciation in quality and quantity with the expressed desire by the students to continue with this form of teaching. Conclusion: While taking into account the experimental and innovating character of this teaching method it seems to have been well received by allowing practical use of learnt theory and attaining the objective of helping the students to become more able on dealing with that unique and specific relationship between a doctor and his patient.
\end{abstract}

Key words Teaching the doctor patient relationship; student rating; teacher rating; scale estimation.

PédagogieM édicale 2004 ; 5 : : 159-166

\footnotetext{
*Département de Formation Médicale Continue et de Développement Pédagogique de la Faculté de médecine de Nantes, 1 rue Gaston Veil, 44035 Nantes Cedex.

* * Service de Médecine Interne A, CHU Hôtel-Dieu, 1 place Alexis Ricordeau, 44091 Nantes cedex 1

Correspondance: Angélique Bonnaud-Département de PMC, Faculté de Médecine de Nantes, 1 rue Gaston Veil, 44035 Nantes Cedex.mailto:angelique.bonnaud@sante.univ-nantes.fr
} 


\section{Concepts et Innovations}

\section{Introduction}

L'introduction des sciences humaines et sociales dans le cursus des études médicales en France pose aujourd'hui la question des contenus à enseigner, des méthodes pédagogiques à privilégier et des évaluations à réaliser. En ce qui concerne les contenus, le thème de la relation médecinpatient est désormais inscrit au programme officiel du deuxième cycle des études médicales en France, dans un module transversal consacré à l'apprentissage de l'exercice médical $^{1}$; ce thème sinsère lui-même dans ce qu'il est convenu d'appeler aujourd'hui le professionnalisme médi$\mathrm{cal}^{2,3}$. L'objectif premier d'un tel enseignement est d'aider les étudiants à appréhender leur future profession dans toute sa complexité, en ne se centrant plus exclusivement sur les aspects biocliniques de la maladie mais en considé rant légitimement le sujet malade. 0 utre le fait que ce nouvel enseignement nécessite des outils et des méthodes pédagogiques appropriés, la question qui se pose est celle de son évaluation. Aussi, dans une dynamique de compréhension et d'ajustement de cet enseignement aux besoins des étudiants, nous avons souhaité nous intéresser à ce qu'en pensent les premiers intéressés, c'est-à-dire les étudiants et les enseignants. Le but de cette étude est de rapporter l'appréciation que les uns et les autres formulaient à l'égard de cet enseignement, tel que nous l'avons développé à la faculté de médecine de $\mathrm{N}$ antes, pour les aspects ayant trait tant à son contenu qu'à l'approche pédagogique mise en œuvre.

\section{Contexte}

Dans un contexte de réforme du deuxième cycle des études médicales, un état des lieux sur l'enseignement/ apprentissage de la relation médecin-patient a éé réalisé à la faculté de médecine de $N$ antes. D e nombreuses données recueillies auprès des responsables facultaires, des enseignants cliniciens (professeur des universités et praticiens hospitaliers), des chefs de clinique et assistants hos pitalo-universitaires, des cadres infirmiers, des étudiants externes ainsi que des résidents en médecine générale ont donné des résultats peu satisfaisants. En effet, l'enseigne ment de la relation médecin-patient ne semblait pas être considéré comme une priorité au regard des contenus scientifiques, biomédicaux et techniques4. C'est ainsi que, faisant suite à une réflexion interdisciplinaire réunissant des médecins, philosophes, psychologues, juristes, etc., une séquence d'enseignement spécifique et formelle a été mise en place au cours de l'année universitaire 2002/2003 et une éval uation auprès des étudiants et des enseignants de cet enseignement a été réalisée.

\section{Problématique pédagogique}

Pour mieux situer l'action évaluative réalisée dans cette étude, il convient de bien distinguer trois grands objets possibles de l'évaluation : l'évaluation des apprentissages, l'évaluation de l'enseignement et celle des enseignants. $D$ ans notre étude, nous avons recueilli, en post-enseignement, l'appréciation des étudiants et des enseignants sur l'enseignement concernant la relation médecin-patient. C ette étude n'a donc pas cherché à évaluer l'efficience de cet enseignement, ni l'impact de ce dernier sur l'acquisition de connaissances, de compétences ou d'habiletés spé cifiques des étudiants, ce qui aurait nécessité l'utilisation de méthodes spécifiques ad hoc plus complexes à mettreen œuvre.

Concernant l'évaluation des enseignements portant soit sur la communication, soit sur la relation médecin-patient incluant l'apprentissage des habiletés de communication, il convient de constater que peu d'études se sont données pour but d'évaluer l'impact de cesenseignements en début de cursus. La majorité a porté sur une évaluation au stade de la formation clinique ${ }^{5}, 6$. En France, encore très peu d'études ont évalué l'impact de ces nouveaux enseigne ments. U ne des premières recherches, réalisée à la faculté demédecine de Rouen par Guigot et coll. en 2002, a évaluél'impact d'un enseignement spécifique sur l'aptitude à la communication orale d'étudiants en médecine de deuxième année'. Cette évaluation réalisée en pré et post enseignement à partir d'entretiens individuels et interactifs, sur la base de quatre items pré-établis, en recourant à un jury de trois enseignants, a permis de montrer de manière significative que les étudiants qui avaient bénéficié de cet enseignement amélioraient rapidement toutes les composantes de leur aptitude à communiquer. Cette étude est à considérer comme une première étape, tant par son contenu que par sa méthode d'évaluation.

D 'une façon générale, les démarches de planification et d'évaluation systématiques des enseignements, concernant notamment l'évaluation de l'impact de ces derniers sur les compétences des éudiants, sont encore peu développées en France, contrairement à de nombreux pays anglosaxons. Le travail de collaboration entre l'université de Montréal et la cité de la santé de Laval au Q uébec, concernant la miseen place d'un enseignement de la communication médecin-patient, est exemplaire à cet égard. 


\section{Évaluation d'un ensegignement concernant la redation médecin-patient...}

Au cours d'ateliers proposés aux étudiants dès la première année et basés sur un modèle précis et complet qui est celui de Calgary-Cambridge, 9, 10, 11, des méthodes d'apprentissages axées sur des jeux de rôle sont utilisées pour favoriser un apprentissage expérientiel des habiletés de communication. Ces séances sont complétées par des séances d'apprentissage par problème et par de rares cours magistraux. La rétroaction, ou feedback, permet de porter un regard critique sur ce qui est fait et de corriger si besoin ces habiletés ${ }^{12}$. C emodèlenous semble intéressant tant par ses méthodes interactives que par une des conditions qu'il satiffait, celle de la continuité sur le cursus, qui en conditionne l'efficacité.

De façon moins ambitieuse et dans le cadre d'une étude exploratoire, notre travail se propose de recueillir les perceptions des étudiants et des enseignants concernés à l'égard du programme de formation mis en place.

\section{Méthodes}

\section{Caractéristiques du dispositif d'enseignement mis en cauvre}

Une séquence d'enseignement formel concernant la relation médecin-patient a été proposée aux étudiants de deuxième (PCEM 2) et de troisième anné (DCEM 1) des études médicales. Pour les deux années, il sagissait d'un enseignement composé de quatre enseignements dirigés et intégrés à un stage permettant une contextualisation. Les enseignants impliqués étaient volontaires, sensibilisés à la dimension de la relation médecin-patient grâce à leur expérience.

Pour les étudiants de deuxième année, il sagissait d'un stage hors hospitalisation de six demi-journées à répartir sur sept semaines chez des médecins libéraux généralistes et spécialistes. Ces derniers avaient reçu pour consigne d'aborder avec l'étudiant tout ce qui se rapportait à la relation médecin-patient. Lesétudiants rapportai ent dansleur carnet de bord les éléments observés et discutés avec leur maitre de stage. Ce stage était complété par quatre enseignements dirigés de deux heures, organisés en début de stage et utilisant des interventions pédagogiques interactives à partir d'activités en sous-groupes; quatre théma tiques étaient abordées : les représentations de la relation médecin-patient en général, lestechniques de base et habiletés de communication, le professionnalisme et l'entrevue médicale.

Pour les étudiants de troisième année, le stage avait lieu dans les services de médecine interne, à raison de trois demi-journées par semaine sur une durée de sept semaines. Les étudiants devaient rencontrer un patient, réaliser un entretien structuré centré sur le patient et rapporter dans leur carnet de bord les éléments de cette rencontre. Ce stage était également complété par quatre enseignements dirigés de deux heures, utilisant également des interventions pédagogiques interactives à partir d'activités en sous-groupes; quatre thématiques différentes étaient abordées: I'approche centrée sur le patient, l'apprentissage du raisonnement éthique, les concepts philosophiques ai dant à la prise de décision éthique et la déontologie médicale.

Pour les deux années, une séance de rétroaction avait lieu après les stages et les enseignements afin de donner aux étudiants la possibilité de s'exprimer sur le vécu de leur stage et de l'enseignement dans sa globalité.

\section{Approche évaluative employée}

D esinformations ont été recueillies respectivement auprès des étudiants et des enseignants. A la fin des séances de rétroaction finale mentionnées plus haut, des questionnaires d'appréciation utilisant uneéchelle de Likert ont été distribués aux étudiants14, 15. Q uatre-vingt-quatre étudiants de deuxième année et soixante et onze étudiants de troisième année ont répondu au questionnaire, soit respectivement 70 et $60 \%$ de chaque promotion concernée. Le questionnaire administré aux étudiants de deuxième année était constitué de quinze questions : quatre questions portai ent sur le fond, c'est-à-dire sur la pertinence de chaque enseignement dirigé par rapport à l'enseignement de la relation médecin-patient; quatrequestions portaient sur la forme, c'est-à-dire sur l'appréciation des étudiants vis-à-vis des méthodes pédagogiques utilisées pour chaque enseignement; trois questions portaient sur l'accueil des étudiants durant leur stage; trois questions portaient sur la possibilité d'observer la relation médecin-patient pendant leur stage et une question concernait l'appréciation global e de l'enseignement.

Le questionnaire administré aux étudiants de troisième année était constitué de treize questions : quatre portaient respectivement sur le fond et sur la forme, comme pour les étudiants de deuxième année ; une question portait sur la motivation des enseignants perçue par les étudiants, une question sur la mise en application du contenu des enseignements dirigés pendant les stages, une question sur la 


\section{Concepts et Innovations}

confrontation à des problèmes d'ordre éthique lors des stages, une question sur la nécessité de poursuivre ce nouvel enseignement et une dernière sur l'appréciation globale de l'enseignement.

L'appréciation des enseignants à l'égard de ces enseigne ments a été recueillie en fin de séquence. Sept des dix enseignants qui ont participé aux enseignements ont répondu au questionnaire. II s'agissait d'un questionnaire qualitatif à réponse ouverte comportant les trois questions suivantes: quels sont les points forts de cet enseignement ? Q uels sont les points faibles de cet enseignement ? Q uelles sont vos propositions pour améliorer cet enseignement? Une démarche d'analyse de contenu a été employée pour traiter le verbatim des réponses.

\section{Résultats}

Les résultats sont présentés par année d'étude, respective ment dans les tableaux I et II. Les résultats obtenus auprès des étudiants sont présentés en pourcentage sous forme de tableaux. Les résultats obtenus auprès des enseignants sont présentés par thématiques sous forme de tableaux.

\section{Discussion}

La principale limite de notre étude tient à son recueil exclusif de l'appréciation du degré de sati faction des étudiants et des enseignants à l'égard des enseignements formels mis en œuvre. La démarche évaluative adoptée ne permet en aucune manière de documenter les habiletés de communication et, plus largement, les compétences relationnelles développées par nos étudiants. Pour cefaire, des travaux complémentaires devront être conduits en utilisant, par exemple, des grilles spécifiques qui ont été déve loppées et validées à cette fin et qui tiennent compte des nombreuses composantes de la communication verbale, non verbale et des principes de base de la relation méde cin-patient ${ }^{13}$.

Les résultats obtenus auprès des étudiants de deuxième année sont globalement satisfaisants (tableau I.1). Les quatre enseignements dirigés sont jugés pertinents du point de vue de leur contenu, surtout en ce qui concerne les enseignements portant sur la sensibilisation à la relation médecin-patient (79\%) et sur l'entrevue médicale $(71,5 \%)$. Les étudiants ont pu êtreguidés au cours deleur stage dans leur observation de la relation médecin-patient respectivement pour $94 \%$ d'entre eux chez les généra- listes, $83 \%$ chez les spécialistes libéraux et $84,5 \%$ chez les hospitaliers. L'accueil reçu par les étudiants lors de leur stage semble avoir été souvent de qual ité avec une appréciation positive pour $96 \%$ des étudiants reçus chez les généralistes, $90 \%$ chez les spécialistes libéraux et $88 \%$ chez les hospitaliers (tableau I.2). Concernant la forme et les méthodes pédagogiquesutilisées, l'appréciation est également positive avec des enseignements jugés « intéressants » sauf pour le cours sur les bases de la communica tion, jugéintéressant par seulement $48 \%$ des éudiants et à l'égard desquels $30 \%$ des étudiantsnese prononcent pas (tableau I.1). Ces premiers résultats quantitatifs indiquent qu'il y a un certain intérêt pour ce nouvel enseignement, tant dans sa forme que dans son contenu, avec une appré ciation globalement positive de $76 \%$, ce qui est incitatif quant à la poursuite de l'enseignement.

Concernant les enseignements de troisième année, l'appréciation des étudiantsn'est nettement positive que pour ceux ayant trait à l'approche centrée sur le patient, tant pour la perception de la pertinence des enseignements dirigés que pour celle de l'intérêt porté aux méthodes pédagogiques utilisées, (respectivement 66 et $61 \%$ d'opinions positives) (tableau II.1) ; cette appréciation est beaucoup plus nuancée pour les enseignements ayant trait à la déontologie, à la décision éthique et à l'apprentissage du raisonnement éthique, avec approximativement autant d'opinions positives que négatives; des études complé mentaires seraient nécessaires pour en comprendre les déterminants. Concernant l'éthique, $54 \%$ des éudiants disent avoir été parfois confrontés à des problèmes éthiques durant leur stage, $15 \%$ disent y avoir été souvent confrontés et $10 \%$ très souvent confrontés (tableau II.2). Ces chiffres peuvent signifier que les étudiants ont été en mesure d'identifier ces problèmes, ce qui est la première étape de l'apprentissage au raisonnement éthique et ce qui témoigne égal ement du développement de leur regard critique. Q uant à la motivation des enseignants perçue par les étudiants, elleest jugée positive par $49 \%$ des étudiants qui pensent que les enseignants sont motivés, $30 \%$ sont plutôt d'accord et seulement 14 \% perçoivent les enseignants comme non motivés (tableau II.3). L'appréciation globale de l'enseignement est moins élevée que pour les étudiants de deuxième année avec une appréciation positive de $58 \%$ d'entre eux ; cependant $73 \%$ des étudiants préconisent la poursuite de cet enseignement pendant le second cycle.

L'évaluation qualitative réalisée auprès des enseignants, tant pour la deuxième que pour la troisième année, 


\section{I - Résultats en deuxième année de médecine.}

Tableau I.1 : Appréciation en pourcentage par les étudiants de $2^{e}$ année (PCEM 2) de la pertinence et de la qualité du contenu des quatre enseignements dirigés

\begin{tabular}{|c|c|c|c|c|c|c|}
\hline Enseignement & Oui/Plutôt oui & $\begin{array}{l}\text { Pertinence } \\
\text { Plutôt non/non }\end{array}$ & NSPP & Intéressant & $\begin{array}{l}\text { Q ualité } \\
\text { Sans intérêt }\end{array}$ & NSPP \\
\hline La relation médecin-patient & 79 & 17 & 5 & 66,5 & 31 & 2,5 \\
\hline Le professionnalisme & 55 & 37 & 8 & 73 & 26 & 1 \\
\hline Bases de la communication & 39 & 31 & 30 & 48 & 22 & 30 \\
\hline L'entrevue médicale & 71,5 & 13 & 15,5 & 76 & 12 & 12 \\
\hline
\end{tabular}

\begin{tabular}{|c|c|c|c|c|c|c|}
\hline \multicolumn{7}{|c|}{$\begin{array}{c}\text { Tableau I.2 : Appréciation en pourcentage par les étudiants de 2e année (PCE M 2) } \\
\text { de l'accuél sur leurs terrains de stage et de la possibilité d'observer la relation } \\
\text { médecin-patient sur ces terrains de stage }\end{array}$} \\
\hline Stage & O ui/Plutôt oui & $\begin{array}{l}\text { Observation } \\
\text { Plutôt non/non }\end{array}$ & NSPP & Insuffisant & $\begin{array}{l}\text { Accueil } \\
\text { Bon/très bon }\end{array}$ & NSPP \\
\hline Généraliste & 94 & 1 & 5 & 1 & 96 & 3 \\
\hline Spécialiste & 83 & 11 & 6 & 7 & 90 & 0 \\
\hline H ospitalier & 84,5 & 11,5 & 4 & 6 & 88 & 5 \\
\hline
\end{tabular}

\section{Tableau I.3 : Appréciation qualitative par les enseignants des aspects positifs et négatifs des} enseignements dispensés en $2^{\mathrm{e}}$ année (PCEM 2)

\begin{tabular}{l|l} 
Points forts du dispositif d'enseignement & Points fai bles du dispositif d'enseignement \\
\hline Participation active des étudiants & Participation « aléatoire » de certains enseignants \\
\hline M éthodes pédagogiques adaptées & O rganisation des stages à revoir \\
\hline Contextualisation des enseignements par les stages & Locaux parfois inappropriés \\
\hline Transversalité des intervenants & M anque d'homogénéité du contenu des enseignements dirigés \\
\hline
\end{tabular}

PCEM 2 : deuxième année du premi er cycle des études médical es 


\section{Concepts et Innovations}

\section{II - Résultats en troisième année de médecine.}

\begin{tabular}{|c|c|c|c|c|c|c|}
\hline Enseignement & O ui/Plutôt oui & $\begin{array}{l}\text { Pertinence } \\
\text { Plutôt non/non }\end{array}$ & NSPP & Intéressant & $\begin{array}{l}\text { Q ualité } \\
\text { Sans intérêt }\end{array}$ & NSPP \\
\hline Approche centrée & 66 & 28 & 6 & 61 & 29 & 10 \\
\hline D éontologie & 25,3 & 32,3 & 42,3 & 28 & 27 & 45 \\
\hline Décision éthique & 34 & 36 & 30 & 36 & 33 & 31 \\
\hline A.R.E. & 39,5 & 25,5 & 35 & 46 & 20 & 34 \\
\hline
\end{tabular}

Tableau II.2 : Appréciation en pourcentage par les étudiants de 3 année (D CEM 1 ) de leur confrontation à des questions d'ordre éthique lors de leur stage

\begin{tabular}{cl}
\hline Confrontation & $\%$ \\
\hline Jamais & 14 \\
\hline Parfois & 54 \\
\hline Souvent & 15 \\
\hline Très souvent & 10 \\
\hline
\end{tabular}

DCEM 1 : première année

du deuxième cyde des études médicales
Tableau II.3 : Appréciation en pourcentage de la motivation des enseignants perçue par les étudiants de $3^{e}$ année (DCEM 1)

\begin{tabular}{lc}
\hline Motivation & $\%$ \\
\hline N SPP & 7 \\
\hline N on & 1 \\
\hline Plutôt pas d'accord & 13 \\
\hline Plutôt d'accord & 30 \\
\hline O ui & 49 \\
\hline
\end{tabular}

NSPP : ne se prononcent pas

DCEM 1 : première année du deuxième cycle des études médicales

\section{Tableau II .4 : Appréciation qualitative par les enseignants des aspects positifs et négatifs des enseignements dispensés en $3^{e}$ année (DCEM 1)}

Points forts du dispositif d'enseignement

Participation active des étudiants

M éthodes pédagogiques adaptées:

apprentissage raisonnement éthique

et carnets de bord

Contextual isation des enseignements

dirigés par les stages
Points faibles du dispositif d'enseignement Participation « aléatoire » de certains enseignants Enseignement de philosophie: peu d'étudiants - intérêt?

L ocaux inappropriés pour les enseignements intégrés au lieu de stage

DCEM 1 : première année du deuxième cycle des éudes médicales 


\section{Évaluation d'un enseignement concernant la redation médecin-patient...}

montre un consensus en ce qui concerne certains points (tableaux 1.3 et 1I.4). Tous rapportent, de manière subjective, une participation active et intéressée des étudiants, ce qui semble être concordant avec ce que rapportent les étudiants. Les enseignants estiment que les méthodes pédagogiques utilisées sont adaptées au contenu des enseignements avec une priorité donnée au travail interactif en petits groupes. Ces derniers pensent également que la contextualisation par les stages devrait permettre une meilleure intégration des données par les étudiants, mêmes cet avis, purement subjectif, n'a pas donné lieu à une évaluation de connaissances ${ }^{16}$. De plus, l'interdisciplinarité et la transversalité des intervenants semblent être une richesse appréciée. Concernant les aspects moins positifs, il semblerait qu'il y ait eu une participation «aléatoire » de certains enseignants ; l'illustration est celle de la séance d'apprentissage au raisonnement éthique qui n'a été proposée qu'à $65 \%$ des étudiants ce qui explique le pourcentage de $35 \%$ de ceux-ci qui ne se sont pas prononcés (tableau II.1). II conviendrait également de revoir l'organisation des stages car certains médecins se sont retrouvés avec plusieurs étudiants et d'autres sans. Une question d'ordre matériel se pose égal ement car les locaux ne sont pas toujours appropriés pour réunir les étudiants, de même qu'un manque de matériel ne permet pas de diversifier les supports pédagogiques. Pour terminer, la nécessité de retravailler l'homogénété des enseignements afin d'éviter les redites et d'assurer une continuité dans la transmission des informations semble capitale.

L'ensemble de ces résultats doit évidemment être appré hendé avec des limites non négligeables. La première d'entreelles est liée aux buts mêmes del'enseignement qui est davantage une sensibilisation à la question de la relation médecin-patient qu'une formation authentiquement dédiée à la construction de compétences. $D$ ans cette dernière perspective, des ressources complémentaires en temps, en locaux, en méthodes pédagogiques appropriées et en enseignants motivés et formés à cette dimension devraient être trouvées. La seconde limite de cette étude, déjà évoquée, porte sur l'échelle d'appréciation, qui de manière succincte a tenté d'appréhender les premières réactions des étudiants sans en comprendre les interprétations. Cette étude confirme qu'il convient d'éviter de réduire l'évaluation de l'enseignement à une simple opération de mesure de satiffaction ${ }^{14}$. C'est pourquoi, il est capital que les prochaines évaluations soient plus amplement développées dans leurs items et puissent éva- luer à la fois le contexte, les intrants et le processus du programme (planification des cours, contenus, bibliographie, matériel, organisation, intérêt des enseignants pour le programme, interaction professeur-étudiant, rétroaction, habiletés d'enseignement... ), mais aussi ses résultats et son impact sur les compétences construites par les étudiants ${ }^{17}$.

\section{Conclusion}

M algré l'ensemble des limites de cette étude, les résultats sont relativement significatifs pour dire que les étudiants accordent une place importante à un enseignement portant sur la relation médecin-patient si celui-ci est intégréà un stage permettant une mise en application, et donc une contextualisation. Les résultats sont également incitatifs quant à la nécessité de poursuivre cet enseignement tout en l'adaptant aux besoins des étudiants et surtout à l'inté rêt et à la faisabilité. L'approche pédagogique qu'il convient de développer pour l'avenir devra à notre sens śinscrire plus radicalement dans le paradigme d'apprentissage, c'est-à-dire privilégier un enchâssement des activités d'enseignement, d'apprentissage et d'évaluation des compé tences à partir de tâches professionnelles authentiques, complexes et complètes au cours des stage ${ }^{18}$.

\section{Remerciements}

N ous exprimons nos remerciements aux étudiants \& aux ensegnants qui ont acceptéde participer à cetteévaluation et dont les résultats vont nous permettre d'améliorer ce nouvel enseignement. 


\section{Concepts et Innovations}

\section{Références}

1. Arrêté du 10 octobre 2000 modifiant l'arrêté du 4 mars 1997 relatif à la deuxième partie du deuxième cyde des études médicales. Journal Officiel de la République Française, n² 241, 17 octobre 2000.

2. Barrier JH, Brazeau-Lamontagne L, Colin R, Q uinton A, Llorca $G$, Ehua $F S$ et le conseil pédagogique de la Conférence internationale des doyens des facultés de médecine d'expression française. La formation au profes sionnalisme des futurs médecins. Recommandations du Conseil Pédagogique de la CIDMEF. Pédagogie M édicale $2004 ; 5$ : 75-81.

3. Leprofessionnalismemédical pour lenouveau millénaire: une charte pour les praticiens Projet pour le professionnalisme médical développé par l'American Board of Internal Medicine (ABIM) Foundation, I'American College of Physicians-American Socisety of Internal Medicine (ACP-ASIM) Foundation et I'European Federation of Internal M edicine (EFIM). Pédagogie M édicale 2004, 5 : 43-45.

4. Côté L. Etat des lieux et orientations de travail sur l'apprentissage de la relation médecin-patient à la faculté de médecine de $\mathrm{N}$ antes Rapport de mission, 2001.

5. Cooper C, M ira M. Who should assess medical student's communication skills : their academic teachers or their patients? M ed Educ 1998 ; 32 : 419-421.

6. Roter, Rosenbaum J, de N egri B, Renaud D, Di Prete Brown $L, H$ ernandez $O$. The effects of a continuing medical education programme in interpersonal satiffaction in Trinidad and Tobago. Med Educ 1998; 32 : 181-189.

7. Guigot M, M enard JF, Weber J, Colin R. M esure de l'impact d'un enseignement spéd fiquesur l'aptitude à la communication orale des étudiants en médecine de $2 \mathrm{e}$ année. Pédagogie M édicale 2002, 3 : 8-13.

8. Kurtz SM, Silverman JD, D raper J. Teaching and learning communication skills in medicine. Oxford : Raddliffe M edical Press 1998.
9. Silverman JD, Kurtz SM and D raper J. Skills for communication with patients Raddiffe M edical Press (0 xford), 1998.

10. Kurtz S, Silverman S, Benson J, D raper J. M arrying content and processin dinical method teaching : enhancing the Clagary-Cambridge Guides Acad M ed 2003 ; $78: 802-809$.

11. http://www.welldosesquareco.uk/trainingtheory/calgary.

12. M illetteB, Lussier M T, Goudreau. L'apprentissagedela communication par les médecins: aspects conceptuels et méthodologiques d'une mission académique prioritaire. Pédagogi e M édicale $2004 ; 5$ : 110-126.

13. Côté L, Savard A, Richard B. Evaluation des compé tences relationnelles du médecin avec le patient. Elaboration et validation d'une grille. Le médecin de famille canadien $2001 ; 47$ : 512-518.

14. N ormand S, Girard M, Koumare K. Evaluation de I'enseignement et des ensignants: de la théorieà la pratique XVe Journée Universitaire Francophone de Pédagogie M édicale, Nancy, 22 au 25 avril 2003.

15. N ormand $S$, Bernard $H$. Les professeurs diniciens se prononcent sur l'évaluation de l'enseignement. Pédagogie M édicale 2002 ; 3 : 202-209.

16. Jouquan J. L'évaluation des apprentissages des étudiants en formation médicale initiale. Pédagogie M édicale $2002 ; 3: 38-51$.

17. Stufflebeam DI, Foley WJ, Gephart WJ, Guba EG, $\mathrm{H}$ ammond $\mathrm{RL}$, M erriman $\mathrm{HO}$, Provus M M. L'évaluation en éducation et la prise de décision. O ttawa : Les éditions N H P (édition française), 1980.

18. Jouquan J, Bail P. A quoi sengaget-on en basculant du paradigme d'enseignement vers le paradigme d'apprentissage? Exemple d'une révision curriculaire en rés danat de médecine générale. Pédagogie M édicale 2003 ; $4: 163-175$. 